\title{
GENETIC FACTORS IN FEBRILE SEIZURES
}

Population based twin panels containing 14,352 twin pairs established in Norway and Virginia were used to study the occurrence of febrile seizures and epilepsy at the Departments of Human Genetics and Neurology, Medical College of Virginia, Virginia Commonwealth University, Richmond, VA; and the Institute of Medical Genetics, Oslo, Norway. There was a history of febrile seizures reported in 257 pairs and a history of epilepsy in 1 or both members of 286 pairs of twins. Virginia twins reported febrile seizures $(1.98 \%)$ slightly more often than Norwegian twins $(1.61 \%)$. Norwegian twin pairs reported epilepsy $(2.19 \%)$ slightly more often than Virginia twin pairs $(1.66 \%)$. There were no significant differences in the concordance rates between Virginian and Norwegian twins for either febrile seizures or epilepsy (for febrile seizures, 0.33 , in monozygotic twins and 0.11 in dizygotic twins; for epilepsy, 0.19 monozygotic and 0.07 dizygotic). The excess of concordant monozygotic twin pairs observed for both epilepsy and febrile seizures was significant and showed a factor of almost 3 to 1 (Corey LA et al. The occurrence of epilepsy and febrile seizures in Virginian and Norwegian twins. Neurology Sept 1991; $41: 1433-1436$ ). (Reprints: Dr. Linda A. Corey, Department of Human Genetics, Medical College of Virginia, Box 33 MCV Station, Richmond, VA 232980033.)

COMMENT. The inheritance of febrile seizures is multifactorial. Siblings have approximately an $8-12 \%$ risk of also having febrile seizures. If the index child and 1 parent are affected, the risks to siblings are $30 \%-40 \%$ (50\% if both parents are affected) (Progress in Pediatric Neurology, Millichap JG ed, Chicago, PNB publishers).

\section{INFANTILE SPASMS}

\section{PET STUDIES OF INFANTILE SPASMS}

Positron emission tomography (PET) was used to determine local cerebral metabolic rates for glucose in 44 infants with spasms at the Departments of Neurology and Pediatrics, Division of Nuclear Medicine and Biophysics and the Brain Research Institute, University of California, Los Angeles, School of Medicine, CA. Ictal events were detected in the EEG in 10 studies during PET. The most consistent abnormality on PET was the symmetrical increase of relative cerebral metabolic rate for glucose in the lenticular nuclei and was apparent in 32 of the 44 infants. This relative hypermetabolism of the lenticular nuclei occurred with both cryptogenic or symptomatic spasms, and was not characterized by any specific EEG abnormality. Every infant with focal abnormalities on CT and/or MRI also had a focal abnormality on PET in the same location, but 17 of 28 infants with focal abnormalities on PET had no detectable focal abnormalities on CT and/or MRI (Chugani HT et al. Infantile spasms: II. Lenticular nuclei and brain stem activation on positron emission tomography. Ann Neurol Jan 1992; 31:212-219). (Correspondence: Dr. Chugani, Division of Pediatric Neurology, Room 22464 MDCC, UCLA School of Medicine, Los Angeles, CA 90024.) 
COMMENT. The lenticular nuclei may contribute to the mechanism of infantile spasms. A cortical-subcortical interaction is proposed in the pathophysiology of infantile spasms. A Commission on Pediatric Epilepsy of the International League Against Epilepsy, following a workshop on infantile spasms, has proposed that the term infantile spasms is too restrictive and the term "spasms" is preferable to designate a special type of epileptic seizure that involves the axial musculature-in flexion, extension or mixed- and that often occurs in clusters. They propose that this type of seizure should be listed in the International Classification of Epileptic Syndromes (Commission 1981) and not be confined to the International Classification of Epileptic Syndromes (Commission 1989).

\section{VALPROATE METABOLITES DURING TREATMENT OF INFANTILE SPASMS}

Metabolite profiles were measured in serum and urine of pediatric patients treated with valproic acid for infantile spasms at the Institute of Toxicology and Embryopharmacology, Free University Berlin and Department of Pediatrics, Rittberg-Krankenhaus, Berlin, Germany. The mean age was 7 months (range 4-12 months) and all 25 patients had the hypsarrhythmia pattern in the EEG. Eighteen patients were seizure free after 3 months of VPA monotherapy in a dose of $100 \mathrm{mg} / \mathrm{kg}$ body weight/day. The main VPA metabolites in serum were the $\beta$-oxidation products (2-en-VPA and 3-ketoVPA) and the major diunsaturated metabolite 2,3'-dien VPA; 4 -en and 3-keto4 -en, 2 potential hepatotoxins, were detected only in very low concentrations. Glucuronide conjugates and the oxidation products represent the most abundant metabolites in urine. Two children had transient abnormal metabolite profiles indicating altered $\beta$-oxidation and associated with hepatomegaly and increased liver enzyme activity (Fisher E et al. Valproate metabolites in serum and urine during antiepileptic therapy in children with infantile spasms: abnormal metabolite pattern associated with reversible hepatotoxicity. Epilepsia Jan/Feb 1992; $\underline{33}: 165-171)$. (Reprints: Professor Dr. H. Nau, Institute of Toxicology and Embryopharmacology, Free University Berlin, Garystrasse 5 , D-1000 Berlin 33, Germany.)

COMMENT. The initial stages of hepatotoxicity reactions to VPA may be accompanied by characteristic changes in VPA metabolism (increased levels of 2-en, 2,3'-dien and 3-en VPA). The concentrations of unsaturated metabolites and enzyme activity became normal in 2 children on reduction of VPA dose and concomitant lowering of fever. These abnormal metabolite patterns occurred predominantly in patients treated with large doses of VPA combined with dexamethasone. The authors propose that the early detection of such abnormal metabolite patterns might decrease the risk of severe hepatic injury by the timely withdrawal of VPA or reduction of dosage.

\section{MRI IN TUBEROUS SCLEROSIS WITH NORMAL I.Q.}

The number, size and distribution of cerebral hamartomas determined by MRI in 11 patients with tuberous sclerosis and normal intellect are 